\title{
Local models of stellar convection
}

\section{The Strouhal number}

\author{
P. J. Käpylä ${ }^{1,2}$, M. J. Korpi ${ }^{3}$, M. Ossendrijver ${ }^{2}$, and I. Tuominen ${ }^{1,3}$
}

\author{
1 Astronomy Division, Department of Physical Sciences, PO Box 3000, 90014 University of Oulu, Finland \\ e-mail: petri.kapyla@oulu.fi \\ 2 Kiepenheuer-Institut für Sonnenphysik, Schöneckstrasse 6, 79104 Freiburg, Germany \\ 3 Observatory, PO Box 14, 00014 University of Helsinki, Finland
}

Received 25 October 2004 / Accepted 9 November 2005

\section{ABSTRACT}

\begin{abstract}
Aims. The Strouhal number ( $\mathrm{St}$ ), which is a nondimensional measure of the correlation time, is determined from numerical models of convection. The Strouhal number arises in the mean-field theories of angular momentum transport and magnetic field generation, where its value determines the validity of certain widely used approximations, such as the first order smoothing (hereafter FOSA). More specifically, the relevant transport coefficients can be calculated by means of a cumulative series expansion if $\mathrm{St}<\mathrm{St}_{\text {crit }} \approx 1$.

Methods. We define the Strouhal number as the ratio of the correlation and turnover times, which we determine separately, the former from the autocorrelation of velocity, and the latter by following test particles embedded in the flow.

Results. We find that the Strouhal numbers are, generally, of the order of 0.1 to 0.4 which is close to the critical value above which deviations from FOSA become significant. Increasing the rotational influence tends to shorten both timescales in such a manner that St decreases. However, we do not find a clear trend as a function of the Rayleigh number for the parameter range explored in the present study.
\end{abstract}

Key words. hydrodynamics

\section{Introduction}

The mean-field theories of angular momentum transport (e.g. Rüdiger 1980, 1989) and hydromagnetic dynamos (e.g. Steenbeck \& Krause 1969; Krause \& Rädler 1980) require knowledge of the Reynolds stresses and the mean electromotive force, respectively. Direct analytical calculation of these quantities for astrophysical purposes is not possible at present due to the lack of an established theory of turbulence. Numerical calculations are not much better off since in most astrophysical systems the computational resources needed in order to resolve all physically relevant scales are several orders of magnitude larger than currently available.

In mean-field theories these problems are circumvented by relating the Reynolds stresses and electromotive force to the mean quantities (the rotation vector $\boldsymbol{\Omega}$ and the mean magnetic field $\langle\boldsymbol{B}\rangle$, respectively) and their gradients by means of a cumulative series expansion (see van Kampen 1974a,b). In the best known and most often used approach, the first order smoothing approximation (FOSA), only the first terms of these expansions are taken into account. This approach can be shown to be valid if either the relevant Reynolds number, $\operatorname{Re}=u l / v, \mathrm{Rm}=u l / \eta$, or the Strouhal number

$\mathrm{St}=\frac{|(\boldsymbol{u} \cdot \nabla) \boldsymbol{u}|}{|\partial \boldsymbol{u} / \partial t|} \approx u \frac{\tau_{\mathrm{c}}}{l_{\mathrm{c}}} \propto \frac{\tau_{\mathrm{c}}}{t_{\mathrm{to}}}$,

is sufficiently small. Above, $u$ and $l$ are the typical velocity and length scales, and $\tau_{\mathrm{c}}$ and $l_{\mathrm{c}}$ are the correlation time and length of the turbulence, respectively. We show in Sect. 3.3 that $1 / t_{\text {to }} \propto u / l_{\mathrm{c}}$. Only the case of small St is relevant in astrophysical circumstances, where typically $\mathrm{Re}, \mathrm{Rm} \gg 1$. Hence the condition St $\ll 1$ should be fulfilled if FOSA is to be valid in stellar convection zones. If this is not the case then FOSA is likely to be too crude a truncation but, as long as St is smaller than the critical value for convergence it is possible to construct higher-order mean-field theories by including more terms of the cumulant expansion. However, a universal critical value of St for convergence of the cumulant expansion does not exist because this depends on the geometry of the problem and on the flow itself. In general though, the critical value is thought to be less than unity. For simple turbulence models it is possible to compute it; Nicklaus \& Stix (1988, hereafter NS88) obtained $\mathrm{St}_{\text {crit }}=1$ for their model. Furthermore, the results of NS88 
show that the higher order effects remain small in comparison to the FOSA result when $\mathrm{St} \lesssim 0.5$.

It is well-known that the requirement for a small Reynolds number is not satisfied in stellar environments. However, the question of the Strouhal number is not settled. On account of the observations of the solar surface granulation, one can estimate the correlation and turnover times to be roughly equal, indicating that $\mathrm{St} \approx 1$ at the solar surface (see e.g. Chap. 6 of Stix 2002). Similar values can also be estimated for supergranulation for which typical numbers are $u \approx 100 \mathrm{~m} \mathrm{~s}^{-1}, l \approx 10^{7} \mathrm{~m}$, and $\tau \approx 10^{5} \mathrm{~s}$. However, even for granulation the precise value has, to our knowledge, not been established, and nothing is known about St in the deeper layers. Furthermore, recent results from forced turbulence calculations indicate that if the higher order correlations in the equations of the passive scalar flux (Brandenburg et al. 2004, hereafter BKM) and electromotive force (Brandenburg \& Subramanian 2005) are taken into account via the so-called minimal $\tau$-approximation (Blackman $\&$ Field 2002, 2003), the Strouhal number can be seen to substantially exceed unity and roughly equal to unity, respectively.

Although forced turbulence is rather different in comparison to convection, the aforementioned studies still raise the question whether the results of the convection calculations can be interpreted within the framework of the standard mean-field theory as has been done in numerous studies during recent years (e.g. Brandenburg et al. 1990; Pulkkinen et al. 1993; Ossendrijver et al. 2001, 2002; Käpylä et al. 2004; Rüdiger et al. 2005). Motivated by the unknown status of the Strouhal number for convection and the previous studies on the subject in different contexts, we set out to calculate St from numerical calculations of convection. In order to do this, we calculate the correlation time from the autocorrelation of velocity and determine the turnover time by following test particles embedded into the flow and define the Strouhal number as the ratio of the two.

The remainder of the paper is organised as follows: Sect. 2 summarises briefly the numerical model used and in Sect. 3 the results of the study are discussed. Finally, Sect. 4 gives the conclusions.

\section{The model}

A detailed description of the convection model can be found in Käpylä et al. (2004, hereafter Paper I) and the convection calculations are made with a setup identical to that used in Paper I. See Table 1 for a summary of the main parameters.

For the purposes of the present study we have added the possibility to follow the trajectories of Lagrangian test particles in the model. In order to integrate the trajectory we need the velocity at the position of the test particle at each integration step. This is done by finding the grid points $\left(n_{x}, n_{y}, n_{z}\right)$ next to the test particle and using linear interpolation to obtain the velocity at the correct position

$\boldsymbol{u}\left(\boldsymbol{x}_{\mathrm{tp}}\right)=\boldsymbol{u}(\boldsymbol{n})-\sum_{i=x, y, z} \frac{\delta x_{i}}{\Delta x_{i}} \boldsymbol{u}\left(\boldsymbol{n}-\boldsymbol{e}_{i}\right)$,

where $\boldsymbol{x}_{\mathrm{tp}}=\left(x_{\mathrm{tp}}, y_{\mathrm{tp}}, z_{\mathrm{tp}}\right)$ is the position vector of the test particle, $\boldsymbol{n}=\left(n_{x}, n_{y}, n_{z}\right)$ denotes the grid point next to the test
Table 1. Summary of the calculations and the main parameters. From left to right: the Rayleigh, Reynolds, Taylor, and Coriolis numbers, the latitude, and the grid size.

\begin{tabular}{lcccccc}
\hline \hline Run & $\mathrm{Ra}$ & $\mathrm{Re}$ & $\mathrm{Ta}$ & $\mathrm{Co}$ & $\Theta$ & Grid \\
\hline lCo0 & $1.25 \times 10^{5}$ & 95 & 0 & 0 & - & $48^{3}$ \\
Co0 & $2.5 \times 10^{5}$ & 140 & 0 & 0 & - & $64^{3}$ \\
$\mathrm{mCo} 0$ & $5.0 \times 10^{5}$ & 190 & 0 & 0 & - & $96^{3}$ \\
$\mathrm{hCo} 0$ & $10^{6}$ & 246 & 0 & 0 & - & $128^{3}$ \\
\hline Co01-00 & $2.5 \times 10^{5}$ & 140 & 203 & 0.10 & $0^{\circ}$ & $64^{3}$ \\
Co01-30 & $2.5 \times 10^{5}$ & 140 & 203 & 0.10 & $-30^{\circ}$ & $64^{3}$ \\
Co01-60 & $2.5 \times 10^{5}$ & 138 & 203 & 0.10 & $-60^{\circ}$ & $64^{3}$ \\
Co01-90 & $2.5 \times 10^{5}$ & 138 & 203 & 0.10 & $-90^{\circ}$ & $64^{3}$ \\
Co1-00 & $2.5 \times 10^{5}$ & 139 & $2.03 \times 10^{4}$ & 1.04 & $0^{\circ}$ & $64^{3}$ \\
Co1-30 & $2.5 \times 10^{5}$ & 145 & $2.03 \times 10^{4}$ & 1.00 & $-30^{\circ}$ & $64^{3}$ \\
Co1-60 & $2.5 \times 10^{5}$ & 141 & $2.03 \times 10^{4}$ & 1.03 & $-60^{\circ}$ & $64^{3}$ \\
Co1-90 & $2.5 \times 10^{5}$ & 139 & $2.03 \times 10^{4}$ & 1.05 & $-90^{\circ}$ & $64^{3}$ \\
Co10-00 & $2.5 \times 10^{5}$ & 337 & $2.03 \times 10^{6}$ & 4.24 & $0^{\circ}$ & $96^{2} \times 64$ \\
Co10-30 & $2.5 \times 10^{5}$ & 121 & $2.03 \times 10^{6}$ & 11.8 & $-30^{\circ}$ & $96^{2} \times 64$ \\
Co10-60 & $2.5 \times 10^{5}$ & 105 & $2.03 \times 10^{6}$ & 13.6 & $-60^{\circ}$ & $96^{2} \times 64$ \\
Co10-90 & $2.5 \times 10^{5}$ & 104 & $2.03 \times 10^{6}$ & 13.7 & $-90^{\circ}$ & $96^{2} \times 64$ \\
\hline
\end{tabular}

particle, $\boldsymbol{e}_{i}$ the unit vector in direction $i, \delta x_{i}=x_{i}-x_{\mathrm{tp}}$ the distance between the test particle and the grid point next to it, and $\Delta x_{i}$ the grid spacing in direction $i$. In the present calculations we follow one thousand test particles that are introduced at the middle of the convectively unstable layer at random horizontal positions after convection has reached a statistically stationary state.

The calculations were made with a modified version of the numerical method described in Caunt \& Korpi (2001). The calculations were carried out on the KABUL and BAGDAD Beowulf clusters at the Kiepenheuer-Institut für Sonnenphysik, Freiburg, Germany, and on the IBM eServer Cluster 1600 supercomputer hosted by CSC Scientific Computing Ltd., in Espoo, Finland.

\section{Results}

\subsection{Correlation time}

We determine the correlation time from the velocity autocorrelation function

$C\left[u_{i}\left(\boldsymbol{x}, t_{0}\right), u_{i}(\boldsymbol{x}, t)\right]=\frac{\left\langle u_{i}\left(\boldsymbol{x}, t_{0}\right) u_{i}(\boldsymbol{x}, t)\right\rangle}{\sqrt{\left\langle u_{i}^{2}\left(\boldsymbol{x}, t_{0}\right)\right\rangle\left\langle u_{i}^{2}(\boldsymbol{x}, t)\right\rangle}}$,

where $i$ denotes the velocity component used, $t_{0}$ and $t$ are the times from which the snapshots were taken, and the brackets denote horizontal averaging. We estimate the correlation time, $\tau_{\mathrm{c}}$, to be the time after which the correlation drops below a fixed threshold value, in this case 0.5 . However, the correlation time still depends on depth, and there are discrete time intervals between the stored snapshots (see Fig. 1). To remedy the latter, we calculate the correlation time within the convection zone for each depth and use linear interpolation to find a more accurate value for the time when $C=0.5$. Furthermore, it makes sense to average the correlation time over the convectively unstable 


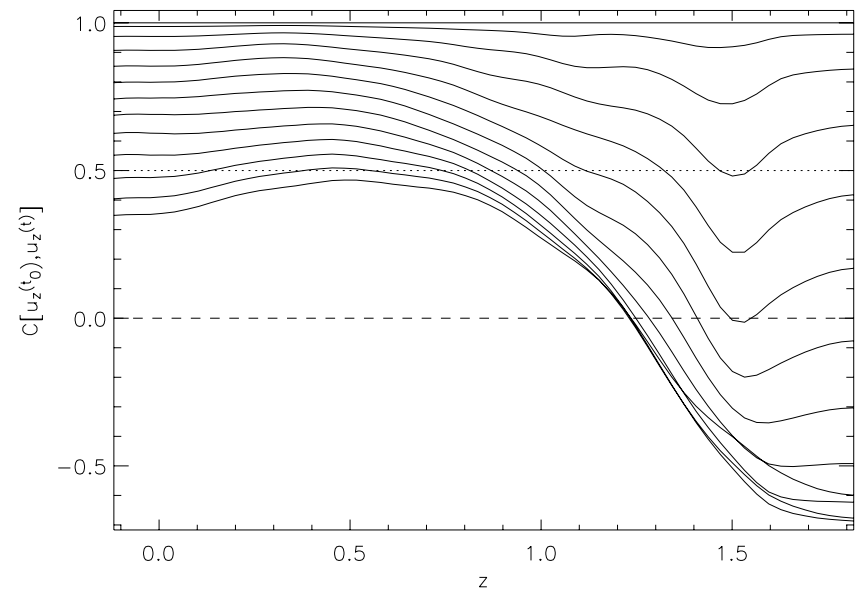

Fig. 1. Horizontally averaged correlations of $u_{\mathrm{z}}$ with respect to the snapshot at $t=100$ for the run Co0. Each curve is separated by $\Delta t=1$ in time units of $\sqrt{d / g}$.

layer since convection is more of a global rather than local nature with the present parameters (see Fig. 3). To check the time dependence of $\tau_{\mathrm{c}}$ we calculate it with respect to about two hundred snapshots for each calculation. The final correlation time is an average of these individual values.

Figure 1 gives an example from run $\mathrm{Co} 0$, showing the horizontally averaged correlations of the vertical velocity $u_{z}$ from twelve snapshots, each separated by one time unit, with respect to the snapshot at $t_{0}=100$. The correlation diminishes monotonically as a function of time and for the eleventh snapshot the correlation is below 0.5 in the whole convection zone. Using the procedure described above, we find the correlation time to be $\tau_{\mathrm{c}} \approx 9.8$ for this snapshot (average over the correlation times with respect to 206 different snapshots gives a value 9.4, see the third column of Table 2). If the correlation is calculated for one of the horizontal velocity components, $\tau_{\mathrm{c}}$ is similar in magnitude, except near the surface and in a layer immediately below the convection zone where it is somewhat longer than the one calculated from the vertical velocity. This effect can be understood to arise from the persistent horizontal flows near the boundaries of the convection zone where the up- and downflows diverge to the horizontal directions. In what follows we estimate the correlation time from the vertical velocity, for which $\tau_{\mathrm{c}}$ remains more or less constant within the convection zone as indicated by Fig. 1. As stated above, our final result is an average over snapshots and the corresponding standard deviations of the correlation times are given in Table 2.

\subsection{On the correlation length}

In principle, the correlation length of the turbulence can be determined from Eq. (3) using $r=\left|x_{1}-x_{2}\right|$ as the argument instead of the time. In order to do this, we choose one thousand random grid points within the convectively unstable layer and compute the crosscorrelations of the velocities between all the points. We do this procedure for the same snapshots from which the correlation times were calculated, and average over all snapshots. Due to the finite spatial resolution of the calculations the smallest distance between two points cannot be

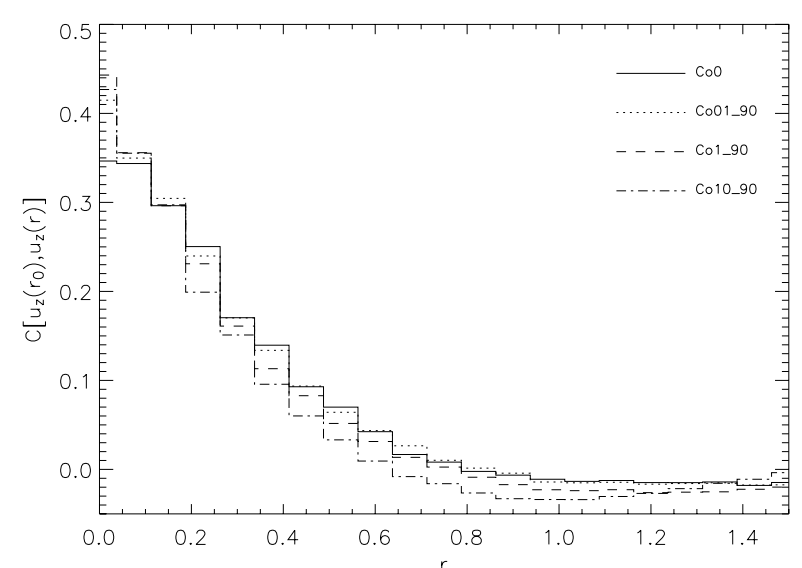

Fig. 2. Velocity autocorrelations for $u_{z}$ as functions of $r$ and rotation for the runs indicated in the legend.

smaller than the grid spacing. Thus the correlations are binned, each bin covering a range $\Delta r=0.075 d$. A characteristic result is shown in Fig. 2, where the correlations from runs with different Coriolis numbers at the southern pole are presented. The main conclusion is that the correlation diminishes faster as a function of $r$ when rotation is more rapid, implying that $l_{\mathrm{c}}$ decreases as a function of rotation. There is, however, a problem of how exactly to define $l_{\mathrm{c}}$. Due to the binning the average correlation in the bin with the smallest $r$ varies from one calculation to the other, and setting a fixed threshold value would be more arbitrary than in the case of the correlation time. We see that a reasonable solution to bypass this problem is to use test particles in order to determine the turnover time, $t_{\mathrm{to}}\left(\propto l_{\mathrm{c}} / u_{\mathrm{t}}\right)$, that captures the changing spatial scale of convection as a function of rotation.

\subsection{Turnover time}

The turnover time is usually estimated by dividing a characteristic length scale by a characteristic velocity. For convection calculations one often uses the depth of the convection zone divided by the volume-averaged rms velocity, i.e.

$t_{\mathrm{to}}^{(\mathrm{s})}=\frac{d}{u_{\mathrm{t}}}$,

where the superscript s refers to "simple estimate". For example, for the Co0 run, $u_{\mathrm{t}} \approx 0.085$ and $d=1$, giving $t_{\mathrm{to}}^{(\mathrm{s})} \approx 12.0$. Using this estimate with $\tau_{\mathrm{c}}=9.4$ would indicate that St is approximately 0.8 . Although this simple method probably gives the correct order of magnitude of the turnover time, it is still quite a crude estimate since the values for the typical scales and velocities are rather uncertain and vary nonlinearly as function of rotation and Rayleigh number (see below). Thus a more precise way of calculation is desirable.

A way to improve the estimate given by Eq. (4) is to follow the trajectories of Lagrangian test particles in the flow. This is done by finding the times where a particle changes its direction, i.e. turns over. Thus, one turnover time would be the time between two consecutive turns to the same direction, e.g. from downward motion to upward motion. The advantage of 


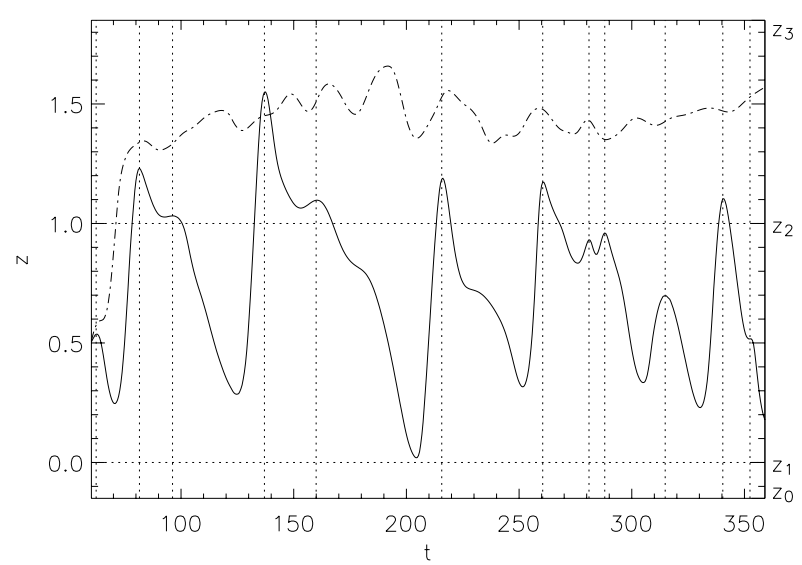

Fig. 3. Vertical positions of two test particles in the run Co0. The dotted vertical lines denote the times where the particle denoted by the solid line changes direction from downward motion to upward motion. The dash-dotted line shows a particle which is stuck in the lower overshoot layer for the whole duration of the calculation.

Table 2. From left to right: rms-velocity averaged over the convectively unstable region and time, correlation time from the autocorrelation of the vertical velocity, turnover time from test particle trajectories, and the Strouhal number. The last column states the number of snapshots with respect to which the correlation times and lengths were calculated.

\begin{tabular}{lccccc}
\hline \hline Run & $u_{\mathrm{t}}$ & $\tau_{\mathrm{c}}$ & $t_{\mathrm{to}}$ & $\mathrm{St}$ & $N$ \\
\hline $\mathrm{CCo} 0$ & 0.085 & $11.2 \pm 2.3$ & 25.9 & 0.43 & 209 \\
$\mathrm{Co} 0$ & 0.084 & $9.4 \pm 2.1$ & 25.1 & 0.37 & 206 \\
$\mathrm{mCo} 0$ & 0.080 & $8.8 \pm 2.6$ & 24.0 & 0.37 & 212 \\
$\mathrm{hCo} 0$ & 0.073 & $8.9 \pm 1.6$ & 23.4 & 0.38 & 199 \\
\hline $\mathrm{Co} 01-00$ & 0.084 & $9.4 \pm 2.2$ & 25.0 & 0.38 & 206 \\
$\mathrm{Co} 01-30$ & 0.084 & $9.2 \pm 1.5$ & 25.1 & 0.37 & 202 \\
$\mathrm{Co} 01-60$ & 0.082 & $9.8 \pm 2.9$ & 25.0 & 0.39 & 207 \\
$\mathrm{Co} 01-90$ & 0.082 & $9.0 \pm 1.6$ & 25.5 & 0.35 & 203 \\
$\mathrm{Co} 1-00$ & 0.083 & $7.3 \pm 1.4$ & 24.5 & 0.30 & 207 \\
Co1-30 & 0.086 & $6.1 \pm 0.9$ & 23.4 & 0.26 & 198 \\
Co1-60 & 0.084 & $6.9 \pm 0.8$ & 23.7 & 0.29 & 207 \\
Co1-90 & 0.083 & $7.1 \pm 0.9$ & 23.8 & 0.30 & 206 \\
Co10-00 & 0.201 & $2.2 \pm 0.3$ & 12.6 & 0.17 & 202 \\
Co10-30 & 0.072 & $3.9 \pm 0.3$ & 21.0 & 0.19 & 200 \\
Co10-60 & 0.062 & $3.4 \pm 0.2$ & 20.5 & 0.17 & 201 \\
Co10-90 & 0.062 & $3.1 \pm 0.4$ & 18.4 & 0.17 & 201 \\
\hline
\end{tabular}

this method is that the assumption of the vertical scale of convection is removed. However, the danger with this method is that the smallest scales begin to dominate due to, for example, contributions from particles stuck in the stable layer. This problem is discussed below. Figure 3 shows the trajectories of two test particles in the run Co0. The solid line shows a particle which is carried by the convective flow throughout the calculation (the particles were introduced in the flow at $t=60$ ). In the figure we only denote the turnovers from downward to upward motion, but including also the opposite changes of direction a total of 21 turnovers are registered. Figure 4 shows a histogram of the registered turnover times from run CoO. In total, the thousand particles make 21300 individual turnovers in the course of the calculation. The distribution is centered near 20 having an exponential tail towards longer times. Arithmetic

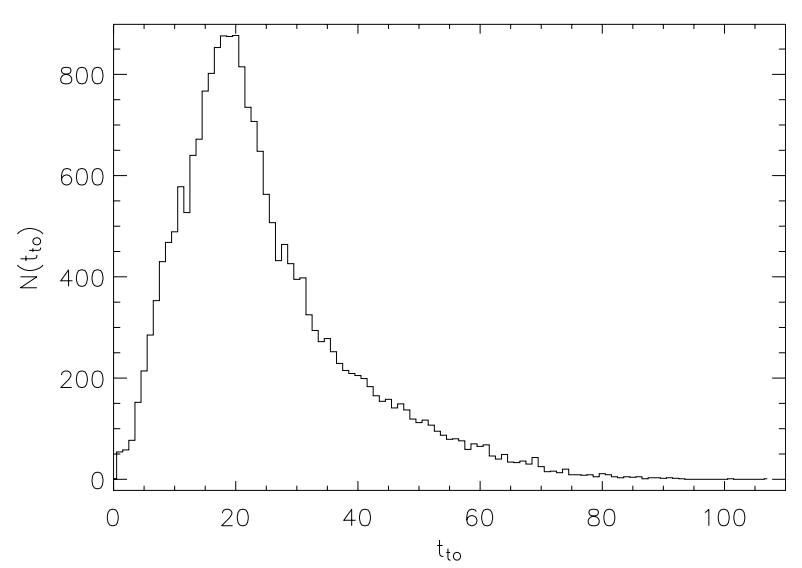

Fig. 4. Distribution of turnover times from run the $\mathrm{Co} 0$ with the test particle method.

average over the distributions shown in Fig. 4 gives a turnover time $t_{\mathrm{to}} \approx 25$, which indicates that the Strouhal number to be significantly less than 0.8 which was obtained with the simple estimate of the turnover time.

The test particle method takes into account the variable spatial scale of convection but it also picks up the small scale turnovers in the stable layer. A small number of particles get stuck in the lower overshoot layer for a long time and some even stay there for the complete duration of the calculation (the dash-dotted line in Fig. 3) and contribute a large number of small turnover times. These particles, however, have only a small effect on the estimate of $t_{\text {to }}$ for the nonrotating and slowly rotating cases where the turnover time might be slightly underestimated.

From Fig. 2 and the second column of Table 2 we can determine that whilst $l_{\mathrm{c}}$ decreases as a function of rotation, a similar trend is visible in $u_{\mathrm{t}}$. Thus we would expect that the ratio $u_{\mathrm{t}} / l_{\mathrm{c}}$ not to vary much as a function of Co. This is indeed the trend that we observe for $t_{\text {to }}$ which seems to support the conjecture that $t_{\mathrm{to}} \propto l_{\mathrm{c}} / u_{\mathrm{t}}$.

\subsubsection{Dependence on the Rayleigh number}

We have made a set of runs varying the Rayleigh number from $1.25 \times 10^{5}$ to $10^{6}$, denoted by $1 \mathrm{Co} 0, \mathrm{Co} 0, \mathrm{mCo} 0$, and $\mathrm{hCo} 0$ in Table 2. With the test particle method $t_{\text {to }}$ decreases slightly as a function of Ra which can be explained by the fact that contributions of smaller scales are now resolved in the model leading to a shorter correlation length, indications of which are also visible in the velocity autocorrelations. However, we do not find any significant trend in the correlation time as function of Ra. It follows that with the present definition the Strouhal number saturates a value approximately 0.4 . This behaviour is similar to the Reynolds number independence of the Strouhal number found by Brandenburg et al. (2004).

\subsubsection{Effects of rotation}

In Paper I, we have shown that the efficiency of convection and overshooting is reduced as rotation increases. The latter 

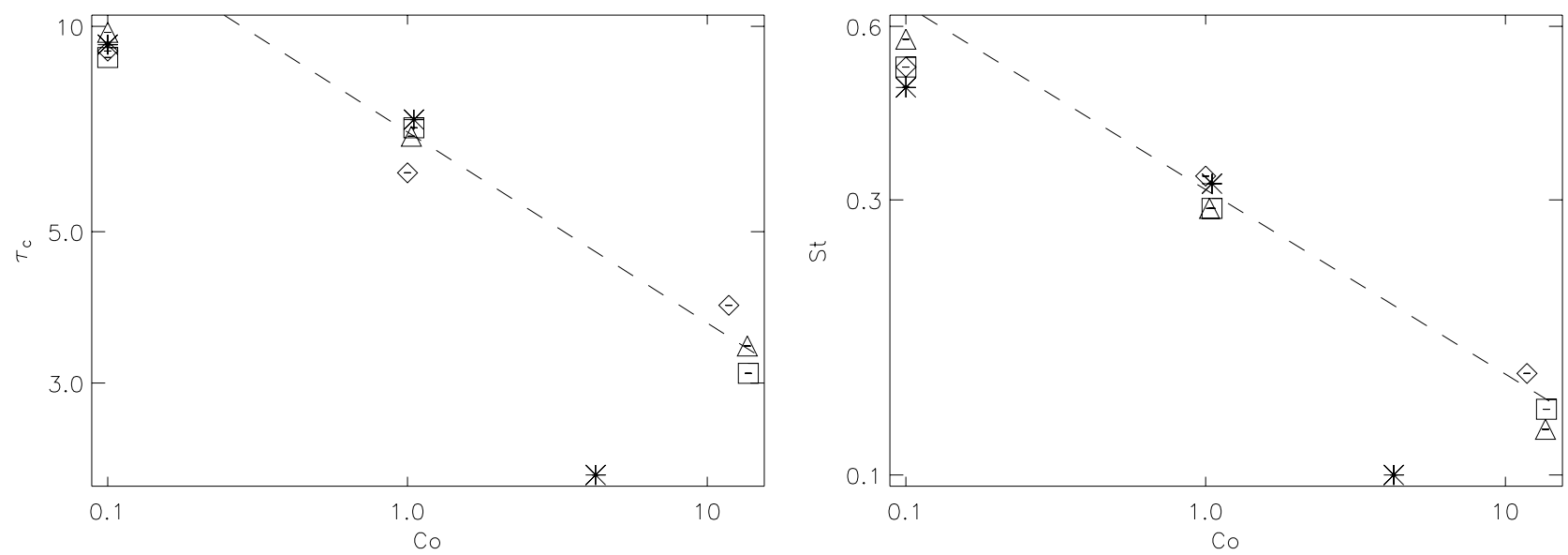

Fig. 5. The correlation time $\tau_{\mathrm{c}}$ (left) and the Strouhal number St (right) functions of the Coriolis number. Powerlaws $\tau_{\mathrm{c}} \propto \mathrm{Co}^{-0.28}$ and St $\propto$ $\mathrm{Co}^{-0.32}$ are plotted. The stars, diamonds, triangles, and squares represents the calculations at latitudes $0^{\circ}$ (equator), $-30^{\circ},-60^{\circ}$, and $-90^{\circ}$ (south pole), respectively.

effect indirectly indicates that the spatial scale of convection decreases, which could lead to shorter turnover time if the overall velocities are less affected. The decreasing horizontal scale of convection as a function of rotation can be seen for example in Fig. 4 of Paper I. In order to study the effects of rotation on the Strouhal number we have made three sets of calculations with varying rotational influence. We denote these runs by the prefix Co01, Co1, and Co10, which correspond to the approximate Coriolis numbers $0.1,1$, and 10 in the runs (the actual values of Co achieved in the calculations vary, see Table 1). We also probe the latitudinal dependence by making calculations at latitudes $0^{\circ},-30^{\circ},-60^{\circ}$, and $-90^{\circ}$ for each rotation rate. The correlation and turnover times, and the resulting Strouhal numbers for all the calculations are presented in Table 2.

For the slowest rotation, we find little differences to the nonrotating case $\mathrm{CoO}$ which was discussed above. Also the variation of the timescales as function of latitude is small in comparison to the variation within individual runs. For the Co1 set the correlation time is markedly shorter (about 6-7 time units as opposed to $9-10$ in the run $\mathrm{Co} 0$ and the $\mathrm{Co} 01$ set). This can be explained by the deflection of the vertical flows by the Coriolis force, leading to smaller vertical scale of convection which tends to shorten the correlation time. Support for this conjecture is given by the decrease of the turnover time from the test particles. A similar decrease in correlation time is noted to occur also if it is estimated from the horizontal velocities.

For the most rapidly rotating case, Co10, the trend of decreasing spatial scales continues. This is manifested in the clearly shorter turnover time $t_{\text {to }}$ as opposed to the more slowly rotating runs discussed above. Here, it is also important to note the misleading value of the turnover time given by the simple method, Eq. (4). Whereas $t_{\mathrm{to}}^{(s)}$ increases with rotation due to the smaller velocities in general, the actual turnover time decreases due to the smaller spatial scale of convection. As for the correlation time, the strong Coriolis forces tend to disrupt the cellular structure of the convection rapidly resulting in a shorter $\tau_{\mathrm{c}}$. Considering the Strouhal number, the decrease in $\tau_{\mathrm{c}}$ overweights the decrease of the turnover time so that the actual value of St decreases as well.
We find that $\mathrm{St}$ is more or less consistent with a powerlaw $\mathrm{St} \propto \mathrm{Co}^{-0.32}$ as a function of the Coriolis number for moderate and rapid rotation (see Fig. 5).

\subsection{Implications for first order smoothing}

The relatively large values of St raise question of the validity of the first order smoothing approximation. To our knowledge, this question has been considered only by NS88, who derive a fourth order correlation approximation from which they derive the transport coefficient responsible for the generation ( $\alpha$-effect) and diffusion of the mean magnetic field for homogenuous and isotropic turbulence for which the velocity field and the correlation time is known. Their results indicate that the fourth order contributions, become comparable to the second order effects, i.e. the FOSA result, if the Strouhal number is of the order 0.5 or larger and that the cumulative expansion fails to converge if St $>1$. In this light our results would indicate that one should be cautious in applying FOSA in the interpretation of the present convection calculations. Direct testing of this, however, requires that we compare analytical mean-field expressions of the relevant transport coefficients to the numerical results. Preliminary results indicate that the correlation time needed to fit the numerical data is significantly shorter than that measured from the velocity autocorrelation function (see Käpylä et al. 2005).

\section{Conclusions}

We estimate the nondimensional measure of the correlation time, the Strouhal number, from numerical models of convection. We calculate the correlation and turnover times separately from the velocity autocorrelation function and the trajectories of embedded test particles, respectively, and define the Strouhal number as the ratio of the two.

The Strouhal number arises in the mean-field theories of angular momentum transport and hydromagnetic dynamos where its value determines the validity of certain widely used approximations, such as the first order smoothing. These 
approximations are based on a cumulative series expansion of the relevant turbulent correlation, e.g. the electromotive force in the dynamo theory. Essentially, the higher order terms in this expansion are proportional to the Strouhal number. Thus the value of St determines whether or not the expansion converges.

The main results can be summarised as follows:

- We find that the correlation time does not depend on the Rayleigh number for the parameter range explored in the present study. Note that the results for $\tau_{c}$ presented here differ significantly from those of Käpylä et al. (2005) due to an error in the analysis in the latter study.

- As function of rotation, $\tau_{\mathrm{c}}$ decreases by a factor of roughly three when the Coriolis number is increased from 0 to 10 . At the same time, the turnover time decreases only by about $20 \%$, leading to an approximate relation $\mathrm{St} \propto \mathrm{Co}^{-0.32}$ for moderate and rapid rotation.

- A noteworthy fact is that if one takes the usual estimate of dividing the characteristic length by the characteristic velocity, the turnover time increases as function of rotation due to the overall decreasing velocities. However, the test particle data gives an opposite result. The discrepancy is due to the fact that even though velocities decrease in general, the spatial scale of convection decreases even more. Thus it is imperative that the definition of St takes into account the spatial scale of the motions, a fact which was also noted by NS88.

Our inability to find any dependence on the Rayleigh number and the relatively high values $(0.1 \ldots 0.4)$ of the Strouhal number raise the question of the validity of FOSA. In the study of NS88, although not from a directly comparable context, the higher order effects became visible when St was of similar magnitude. At the moment, we cannot draw a firm conclusion whether or not it is safe to use FOSA or not. We think that the next step in the direction of determining this would be to develop mean-field expressions for the transport coefficients that are directly applicable to the present numerical calculations. Furthermore, these expressions should be derived using FOSA and preferably also with some higher order expansion in order to determine which of them gives a better description of the numerical result, and, more importantly, whether the whole concept of the cumulative expansion is applicable. This study, however, does not fit in the scope of the present paper.
Acknowledgements. P.J.K. acknowledges the financial support from the Finnish graduate school of astronomy and space physics and travel support from the Kiepenheuer intitute. P.J.K. thanks NORDITA and its staff for their hospitability during his visit. M.J.K. acknowledges the hospitality of LAOMP, Toulouse and the KiepenheuerInstitut, Freiburg during her visits, and the Academy of Finland project 203366. Travel support from the Academy of Finland grant 43039 is acknowledged. The authors thank Axel Brandenburg and Michael Stix for their useful comments on the manuscript and Wolfgang Dobler for illuminating discussions. The anonymous referee is acknowledged for the helpful comments on the manuscript.

\section{References}

Blackman, E. G., \& Field, B. G. 2002, Phys. Rev. Lett., 89, 265007 Blackman, E. G., \& Field, B. G. 2003, Phys. Fluids, 15, L73

Brandenburg, A., Tuominen, I., Nordlund, Å., Pulkkinen, P., \& Stein, R. F. 1990, A\&A, 232, 277

Brandenburg, A., Käpylä, P. J., \& Mohammed, A. 2004, Phys. Fluids, 16,1020

Brandenburg, A., \& Subramanian, K. 2005, A\&A, 439, 835

Caunt, S. E., \& Korpi, M. J. 2001, A\&A, 369, 706

Chan, K. L. 2001, ApJ, 548, 1102

Käpylä, P. J., Korpi, M. J., \& Tuominen, I. 2004, A\&A, 422, 793 (Paper I)

Käpylä, P. J., Korpi, M. J., Ossendrijver, M., \& Tuominen, I. 2005, AN, 326, 186

Krause, F., \& Rädler, K.-H. 1980, Mean-Field Electrodynamics and Dynamo Theory (Berlin: Akademie-Verlag)

Nicklaus, B., \& Stix, M. 1988, Geophys. Astrophys. Fluid Dynamics, 43, 149 (NS88)

Ossendrijver, M., Stix, M., \& Brandenburg, A. 2001, A\&A, 376, 726

Ossendrijver, M., Stix, M., Brandenburg, A., \& Rüdiger, G. 2002, A\&A, 394, 735

Pulkkinen, P., Tuominen, I., Brandenburg, A., Nordlund, Å., \& Stein, R. F. 1993, A\&A, 267, 265

Rüdiger, G. 1980, Geophys. Astrophys. Fluid Dyn., 16, 239

Rüdiger, G. 1989, Differential rotation and stellar convection: Sun and solar-type stars (Berlin: Akademie-Verlag)

Rüdiger, G., Egorov, P., Kitchatinov, L. L., \& Küker, M. 2005, A\&A, 431,345

Steenbeck, M., \& Krause, F. 1969, AN, 291, 49

Stix, M. 2002, The Sun: An introduction, Second edition (Berlin, Heidelberg, New York: Springer-Verlag)

van Kampen, N. G. 1974a, Physica, 74, 215

van Kampen, N. G. 1974b, Physica, 74, 239 